$03,10,19$

\title{
Влияние фокусировки на распространение фононов и теплопроводность в монокристаллических пленках с различным типом анизотропии упругой энергии
}

\author{
(С) И.И. Кулеев
}

Институт фризики металлов им. М.Н. Михеева УрО РАН, Екатеринбург, Россия

E-mail: kuleyevll@imp.uran.ru

(Поступила в Редакцию 15 марта 2017 г.

В окончательной редакции 29 ноября 2017 г.)

\begin{abstract}
Исследовано влияние анизотропии упругой энергии на распространение фононов и фононный транспорт в монокристаллических нанопленках с различным типом анизотропии упругой энергии в режиме кнудсеновского течения фононного газа. Проанализировано распределение длин пробега фононов по углам в плоскостях пленок и в поперечном сечении. Изучены физические причины, приводящие к зависимости теплопроводности от ориентации плоскостей пленок и направлений потока тепла относительно осей кристалла. Анализ влияния фокусировки на распространение фононов позволил объяснить качественное отличие анизотропии длин пробега фононов в пленках из кубических нанокристаллов различного типа, имеющих разную ориентацию плоскостей.
\end{abstract}

Работа выполнена в рамках государственного задания ФАНО России (тема „Спин“ № 01201463330).

DOI: 10.21883/FTT.2018.05.45779.077

\section{1. Введение}

Интерес к исследованию фононного транспорта в нанопленках связан с широким использованием их в микроэлектронике [1-5]. Рассеяние фононов на границах пленок играет важную роль в теплосопротивлении в интервале температур от гелиевых до комнатных [6-8]. Экспериментальные исследования теплопроводности кремниевых нанопроводов диаметром от 22 до $115 \mathrm{~nm}$ были проведены в работе [1], и в кремниевых пленках с толщинами от 20 до $1600 \mathrm{~nm}$ в работах [2-4]. Эти данные по теплопроводности пленок и нанопроводов интерпретировались в модели изотропной среды. При изложении экспериментальных результатов в работах [1-4] не учитывались эффекты, обусловленные фокусировкой фононов: не указывались ориентации плоскостей пленок относительно кристаллографических осей (см. также обзор [5]). Это приводило к погрешности 1.7 раз для кремниевых нанопроводов и в 2.5 раза для теплопроводности кремниевых пленок, поскольку не учитывалась зависимость теплопроводности от направления потока тепла в нанопроводах и от ориентаций плоскостей пленок (см. работы [7-8]). В [7-8] было показано, что граничное рассеяние фононов вносит существенный вклад в теплопроводность достаточно тонких пленок даже при комнатных температурах (согласно [8], более $50 \%$ ).

В настоящей работе проанализируем влияние анизотропии упругой энергии на распространение и рассеяние фононов в монокристаллических пленках из кубических кристаллов с различным типом анизотропии упругой энергии в режиме граничного рассеяния.
В работе [9] показано, что влияние анизотропии упругой энергии на спектр и вектора поляризации колебательных мод определяется безразмерным параметром $k-1=\left(c_{12}+2 c_{44}-c_{11}\right) /\left(c_{11}-c_{44}\right)\left(c_{i j}-\right.$ упругие модули второго порядка). В зависимости от знака параметpa $k-1$, согласно [9], все кубические кристаллы могут быть разделены на кристаллы с положительной $k-1>0$ $(\mathrm{LiF}, \mathrm{GaSb}, \mathrm{GaAs}$ и $\mathrm{GaN})$ и отрицательной $k-1<0$ $\left(\mathrm{CaF}_{2}, \mathrm{SrF}_{2}\right)$ анизотропией упругих модулей второго порядка (см. табл. 1). Значения упругих модулей второго порядка взяты из работ [10-14]. Для изотропных сред параметр $k-1=0$. Проведенный в работе [10] анализ показал, что направления фокусировки колебательных мод в кристаллах первого типа соответствуют направлениям дефокусировки в кристаллах второго типа [10].

Особенности фононного транспорта в наноструктуpax в режиме граничного рассеяния рассматривались в работах $[10,16]$. В [10], главным образом, анализиро-

Таблица 1. Упругие модули второго порядка $c_{i j}$ $\left(10^{12} \mathrm{dyn} / \mathrm{cm}^{2}\right)$, плотность $\rho\left(\mathrm{g} / \mathrm{cm}^{3}\right)$ и параметр анизотропии $k-1$ для рассматриваемых кубических кристаллов. (Данные взяты из работ [11-15])

\begin{tabular}{c|c|l|l|l|l|l}
\hline Тип & Кристалл & $c_{11}$ & $c_{12}$ & \multicolumn{1}{c}{$c_{44}$} & \multicolumn{1}{c}{$\rho$} & $k-1$ \\
\hline \multirow{4}{*}{$\mathrm{I}$} & $\mathrm{LiF}^{c}$ & 1.246 & 0.424 & 0.649 & 2.646 & 0.78 \\
& $\mathrm{GaSb}^{b}$ & 0.885 & 0.404 & 0.433 & 5.62 & 0.85 \\
& $\mathrm{GaAs}^{a}$ & 1.1904 & 0.5384 & 0.5952 & 5.317 & 0.90 \\
& $\mathrm{GaN}$ & 2.93 & 1.59 & 1.55 & 6.15 & 1.275 \\
\hline \multirow{2}{*}{$\mathrm{II}$} & $\mathrm{SrF}_{2}$ & 1.644 & 0.502 & 0.347 & 3.18 & -0.204 \\
& $\mathrm{CaF}_{2}^{h}$ & 1.74 & 0.56 & 0.359 & 3.211 & -0.33
\end{tabular}


валось изменение анизотропии теплопроводности наноструктур при переходе от нанопроводов к квадратным $(L=W=100 D, D=50 \mathrm{~nm}$ ) пленкам (длина которой $L$ равна ширине пленки $W$ ) с различной ориентацией плоскостей. В работе [16] исследовался фононный транспорт в пленках на основе кристаллов первого типа $\mathrm{Ge}$, $\mathrm{Si}$, алмаза. Настоящая работа является продолжением этих исследований. Основное внимание в ней уделено исследованию влияния фокусировки на распространение фононов и фононный транспорт в пленках с различным типом анизотропии упругой энергии. Проанализировано влияние анизотропии упругой энергии на распределение потока фононов как в плоскостях пленок, так и в перпендикулярном сечении. Показано, что оно приводит к качественному отличию анизотропии длин пробега фононов в пленках из кубических кристаллов различного типа. Исследования этих проблем являются важными для технического использования пленок в микроэлектронике.

\section{2. Влияние фокусировки фононов на анизотропию теплопроводность в квадратных пленках с различной ориентацией плоскостей}

Рассмотрим влияние анизотропии упругой энергии на кнудсеновское течение фононного газа в пленках, когда теплосопротивление обусловлено диффузным рассеянием фононов на границах. Проанализируем влияние фокусировки фононов на длины пробега фононов в квадратных пленках $(L=W)$ с различным типом анизотропии упругой энергии для трех случаев, когда направление теплового потока вращается в плоскости пленки и совпадает: (1) с плоскостью грани куба $Y Z\{J\}=\{100\},(2)$ с диагональной плоскостью $\{J\}=\{110\}$, (3) с плоскостью перпендикулярной диагонали куба $\{J\}=\{111\}$. Согласно $[10,16]$, анизотропия теплопроводности и вкладов в нее от различных колебательных мод определяется длинами свободного пробега $\Lambda_{[I(\psi)]}^{\{J\}}$ и $\Lambda_{[I(\psi)]}^{\lambda\{J\}}$, которые зависят от двух ориентационных параметров: направления потока тепла $[I(\psi)]$ и ориентации плоскости пленки $\{J\}$. Исследуем физические причины, приводящие к анизотропии длин пробега фононов, и сравним с результатами, следующими из модели изотропной среды, которая является удобной системой сравнения для длин пробега в упруго анизотропных пленках, поскольку они полностью определяются геометрическими параметрами [10].

Рассмотрим сначала особенности фононного транспорта в квадратных пленках (см. рис. 1). При сравнении угловых зависимостей длин свободного пробега фононов в пленках с различным типом упругой анизотропии следует обратить внимание на следующие особенности. Вопервых, угловые зависимости длин свободного пробега фононов в пленках из кристаллов обоих типов с ориентациями плоскостей $\{001\}$ и $\{111\}$ изотропны, тогда как для $\{J\}=\{110\}$ имеют эллипсоидальный вид. Причем, для $\{J\}=\{110\}$ в кристаллах первого типа длинная ось направлена вдоль [100], для кристаллов второго типа вдоль [110]. Во-вторых, в пленках из кристаллов первого типа с ориентациями плоскостей $\{001\}$ средние длины пробега для всех материалов принимают максимальные значения и оказываются больше, чем в модели изотропной среды (см. рис. $1, a)$, тогда как в пленках из кристаллов второго типа они принимают минимальные значения и оказываются меньше, чем в модели изотропной среды (см. рис. $1, d)$. Так, например, для $\mathrm{GaAs}$ они превосходят $\Lambda_{i s o}$ в 1.9 , а для $\mathrm{CaF}_{2}$ они на $11 \%$ меньше, чем $\Lambda_{i s o}$. В-третьих, для пленок с ориентациями плоскостей $\{111\}$ складывается обратная ситуация: в пленках из кристаллов первого типа средние длины пробега оказываются меньше, а в пленках из кристаллов второго типа больше, чем $\Lambda_{i s o}$ (см. рис. $\left.1, c, f\right)$. Так, для пленок GaAs они становятся меньше, чем $\Lambda_{i s o}$ на $12 \%$, а для пленок $\mathrm{CaF}_{2}$ они становятся на $18 \%$ больше $\Lambda_{i s o}$. Итак, мы показали, что анизотропия длин свободного пробега в квадратных пленках для кристаллов первого и второго типа качественно отличается.

Далее проанализируем влияние упругой анизотропии на распространение акустических мод в квадратных пленках с различной ориентацией и определим физические причины, которые обуславливают зависимость теплопроводности от ориентации плоскостей пленок. Для пленок с плоскостью $\{100\}$ медленная квазипоперечная мода $t_{2}$ фокусируется и дефокусируется в направлениях [100] и [110] соответственно. Для продольных фононов в этих направлениях происходит дефокусировка и локальный максимум фокусировки соответственно. Угол между этими направлениями составляет $\Delta \varphi=\pi / 4$. В пленках с плоскостью $\{111\}$ угол между направлениями фокусировки [110] и дефокусировки [11 $\overline{2}]$ для моды $t_{2}$ и продольных фононов составляет $\Delta \varphi=\pi / 6$.

Рассмотрим распределение длин пробега фононов в пленках с различной ориентацией по углам $\Phi(\theta, \varphi)$ в плоскости пленок, а также их распределение по углам $\Theta(\theta, \varphi)$ в поперечных сечениях. Для этого перейдем в систему координат, связанную с пленкой. Ось $Z$ направим перпендикулярно плоскости пленки. В этом случае угол $\Phi$ определяет распределение теплового потока в плоскости пленки, а угол $\Theta-$ в поперечном сечении. В новой системе координат распределение длин пробега в пленках по углам $\Theta$ и $\Phi$ может быть определено следующим образом:

$$
\begin{gathered}
\Lambda_{[I]}^{\lambda\{J\}}(\Theta)=\frac{1}{2 \pi} \int_{0}^{2 \pi} d \Phi \Lambda_{[I(\psi)]}^{\lambda\{J\}}(\Theta, \Phi), \\
\Lambda_{[I]}^{\lambda\{J\}}(\Phi)=\frac{1}{2} \int_{-1}^{1} d(\cos \Theta) \Lambda_{[I(\psi)]}^{\lambda\{J\}}(\Theta, \Phi) .
\end{gathered}
$$

В пленках, имеющих ориентацию $\{100\}$ угол $\Phi=\varphi$ и $\Theta=\theta$, а величины $\Lambda_{[I(\psi)]}^{\lambda\{J\}}(\theta, \varphi)$ определены в [16]. 

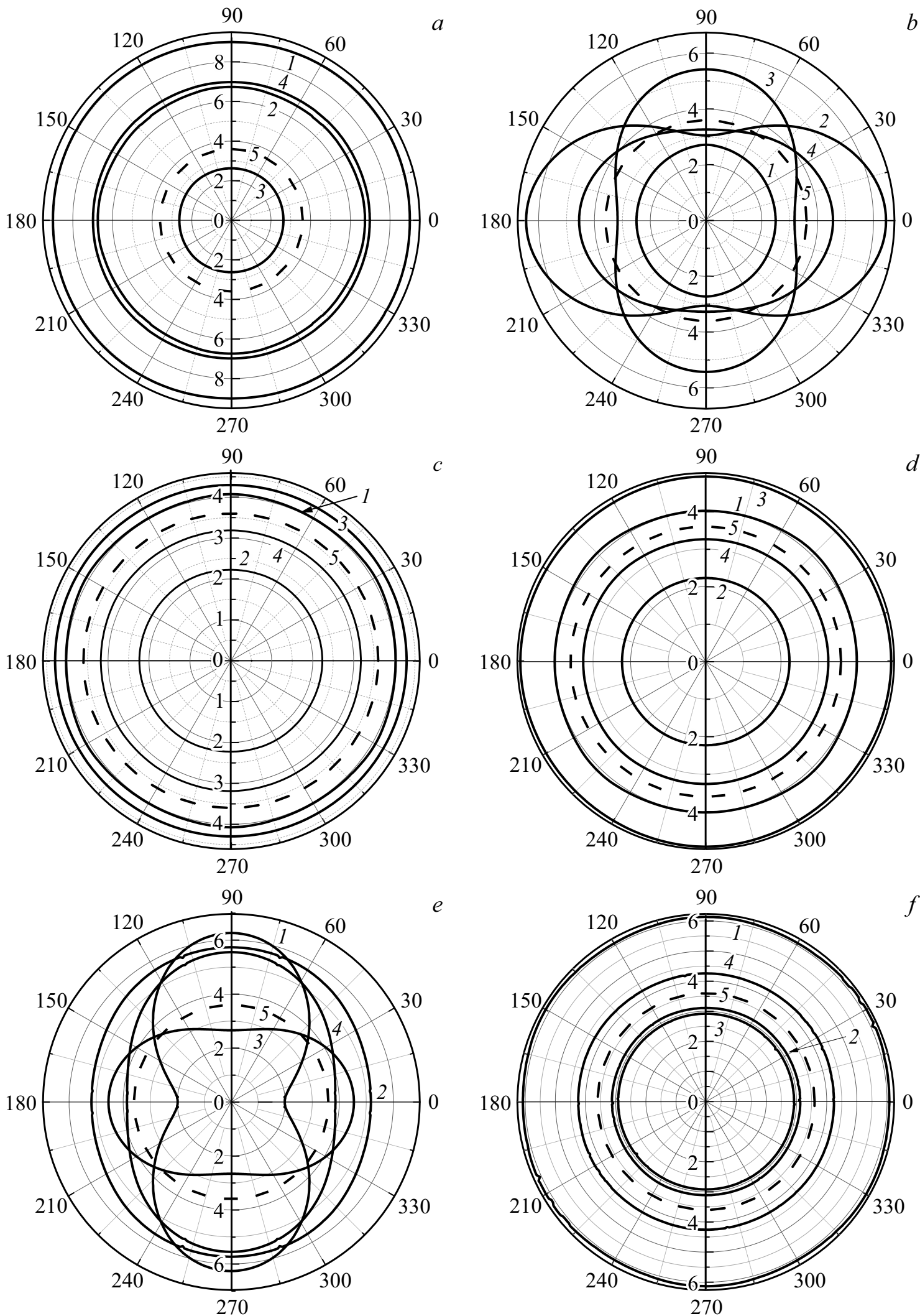

Рис. 1. Угловые зависимости длин свободного пробега фононов $\tilde{\Lambda}_{[I(\psi)]}^{\lambda\{J\}}=\Lambda_{[I(\psi)]}^{\lambda\{J\}} / D$ и $\tilde{\Lambda}_{[I(\psi)]}^{\{J\}}=\Lambda_{[I(\psi)]}^{\{J\}} / D$, нормированных на толщину квадратных пленок с параметрами $L=W=100 D$ и $D=50 \mathrm{~nm}$, для $\mathrm{GaAs}(a, b, c)$ и $\mathrm{CaF}_{2}(d, e, f)$, когда градиент температур вращается в плоскости пленки $\{100\}(a, d),\{110\}(b, e)$ и $\{111\}(c, f)$. Кривые 1 - быстрая поперечная мода, $2-$ медленная поперечная мода, 3 - продольная мода, 4 - средняя длина свободного пробега, 5 - длина свободного пробега, рассчитанная в модели изотропной среды. 
Таблица 2. Отношения длин свободного пробега для квадратных пленок с параметрами $L=W=100 D$ и $D=50 \mathrm{~nm}$ в симметричных направлениях

\begin{tabular}{c|c|c|c}
\hline Соединение & $\Lambda_{[I(\psi)]}^{t 1\{100\}}: \Lambda_{[I(\psi)]}^{t 2\{100\}}: \Lambda_{[I(\psi)]}^{L\{100\}}: \bar{\Lambda}_{[I(\psi)]}^{\{100\}}: \Lambda_{i s o}$ & $\Lambda_{[I(\psi)]}^{t 1\{11\}}: \Lambda_{[I(\psi)]}^{t 2\{111\}}: \Lambda_{[I(\psi)]}^{L\{111\}}: \bar{\Lambda}_{[I(\psi)]}^{\{100\}}: \Lambda_{i s o}$ & $\Lambda_{[100]}^{\{100\}}: \Lambda_{[100]}^{\{110\}}: \Lambda_{[110]}^{\{110\}}: \Lambda_{[110]}^{\{111\}}$ \\
\hline $\mathrm{LiF}$ & $3.36: 1.84: 0.76: 2.23: 1$ & $1.13: 0.65: 1.18: 0.91: 1$ & $2.45: 1.51: 1.04: 1$ \\
$\mathrm{GaSb}$ & $2.57: 1.87: 0.74: 1.97: 1$ & $1.12: 0.62: 1.19: 1$ & $2.23: 1.44: 1.03: 1$ \\
$\mathrm{GaAs}$ & $2.50: 1.87: 0.73: 1.94: 1$ & $1.12: 0.62: 1.19: 0.89: 1$ & $2.20: 1.44: 1.03: 1$ \\
$\mathrm{GaN}$ & $1.85: 1.86: 0.68: 1.7: 1$ & $1.07: 0.64: 1.22: 0.86: 1$ & $1.97: 1.35: 1.04: 1$ \\
$\mathrm{SrF}_{2}$ & $1.01: 0.73: 1.17: 0.9: 1$ & $1.52: 0.91: 0.9: 1.16: 1$ & $0.78: 1.06: 1.61: 1$ \\
$\mathrm{CaF}_{2}$ & $1.12: 0.62: 1.37: 0.9: 1$ & $1.69: 0.87: 0.81: 1.18: 1$ & $0.77: 0.91: 1.31$
\end{tabular}

Определим актуальный интервал углов, дающий основной вклад при усреднении длин пробега по углам $\Phi$ в плоскости пленки. Для этого построим зависимости длин пробега от угла $\Phi$ и определим актуальный интервал углов, дающий основной вклад в $\Lambda_{[I(\psi)]}^{\lambda\{J\}}(\Phi)$. Анализ показал, что для квадратных пленок этот интервал углов составляет приближенно $\Delta \Phi \cong \pi / 2$, а для некоторых мод несколько превышает это значение. Из этого следует, что для произвольного направления потока тепла область усреднения захватывает одновременно направления фокусировки и дефокусировки фононов. Поэтому после усреднения по углам Ф для квадратных пленок с ориентациями $\{100\}$ и $\{111\}$ длины пробега становятся изотропными (см. рис. 1).

Как видно из рис. 1, $a$, для квадратных пленок на основе кристаллов первой группы с ориентацией $\{100\}$ максимальную длину свободного пробега имеют быстрые поперечные фононы, для них $\Lambda_{[I(\psi)]}^{t 1\{100\}}$ оказывается больше, чем в модели изотропной среды. Так, например, для пленок GaAs отношение $\Lambda_{[I(\psi)]}^{t 1\{100\}}: \Lambda_{i s o}$ составляет 2.5. Длины пробега продольных фононов для $\{J\}=\{100\}$ имеют значения меньшие, чем $\Lambda_{i s o}: \Lambda_{[I(\psi)]}^{L\{111\}}: \Lambda_{i s o}=0.73$. В противоположность этому в квадратных пленках на основе кристаллов второй группы с ориентацией $\{100\}$ максимальную длину свободного пробега имеют продольные фононы: для пленок $\mathrm{CaF}_{2} \Lambda_{[I(\psi)]}^{L\{100\}}: \Lambda_{i s o}=1.4$. А минимальную - медленные поперечные фононы: для $\mathrm{CaF}_{2} \Lambda_{[I(\psi)]}^{t 2\{100\}}: \Lambda_{\text {iso }}=0.62$. Для остальных кристаллов отношения длин свободного пробега для симметричных направлений приведены в табл. 2.

При переходе к пленкам с $J=\{111\}$ в кристаллах первого типа длины пробега поперечных фононов уменьшаются более, чем в 2 раза: при этом, для медленной поперечной моды они становятся меньше $\Lambda_{i s o}$ : для GaAs $\Lambda_{[I(\psi)]}^{t 2\{11\}}: \Lambda_{i s o}=0.62$. Напротив, длины пробега для продольных фононов возрастают и становятся в 1.2 раза больше, чем $\Lambda_{i s o}$. А для пленок из кристаллов второго типа длины пробега поперечных фононов увеличиваются, а продольных уменьшаются. Максимальные длины пробега имеет быстрая поперечная мода: для $\mathrm{CaF}_{2}$ $\Lambda_{[I(\psi)]}^{t 1\{100\}}$ в 1.7 раза больше, чем $\Lambda_{i s o}$. Длины пробега для продольных фононов становятся меньше, чем $\Lambda_{i s o}$ : так для $\mathrm{CaF}_{2} \Lambda_{\text {iso }}=0.8$.

Другая ситуация с анизотропией имеет место для потока тепла в квадратных пленках с $\{J\}=\{110\}$, где зависимости теплопроводности и средних длин пробега имеют эллипсоидальный вид с длинной осью в направлении [100] для кристаллов первого типа, и в направлении [110] для кристаллов второго типа. Как видно из рис. $1, b$, для кристаллов первого типа эта зависимость обусловлена модой $t_{2}$, которая фокусируется в направлении [100] и определят направление длинной оси эллипса. Направление ее дефокусировки [110] определяет короткую ось эллипса. Для пленок из кристаллов второго типа анизотропия длин свободного пробега определяется быстрой поперечной модой, которая фокусируется и дефокусируется в направлениях [110] и [100] соответственно. Таким образом, при переходе к пленкам из кристаллов второго типа направления длинной и короткой осей эллипса меняются местами (см. рис. $1, e)$. Однако угол между этими направлениями в обоих случаях составляет $\Delta \Phi=90^{\circ}$. Этот угол оказывается слишком велик, чтобы при усреднении по углу $\Phi$ в плоскости пленки полностью размыть эффект фокусировки, в отличие от пленок с $\{J\}=\{100\}$ и $\{111\}$. Поэтому при усреднении по углам $\Phi$ анизотропия вкладов фононных мод в теплопроводность не усредняется, и теплопроводность в плоскости пленки $J=\{110\}$ остается анизотропной, как и вклады поперечных и продольных фононов (см. рис. 1,c,e). Для медленной поперечной моды длина пробега в пленках GaAs в направлении [100] оказывается в 1.8 раза больше, чем в модели изотропной среды. Однако в направлениях дефокусировки она становится меньше на 10\%, чем в изотропной среде. Для продольных фононов имеем обратную ситуацию: их длины пробега в пленках GaAs в направлении [110] оказываются в 1.5 раза больше, а в направлении [100] — в 1.4 меньше, чем $\Lambda_{i s o}$. Однако в виду малой по сравнению с поперечными фононами плотностью состояний их вклад недостаточен, чтобы кардинально изменить вид полной теплопроводности для рассматриваемых материалов.

Проведенный анализ показал, что с увеличением длины пленок с $J=\{100\}$ и $\{111\}$ интервалы углов, 

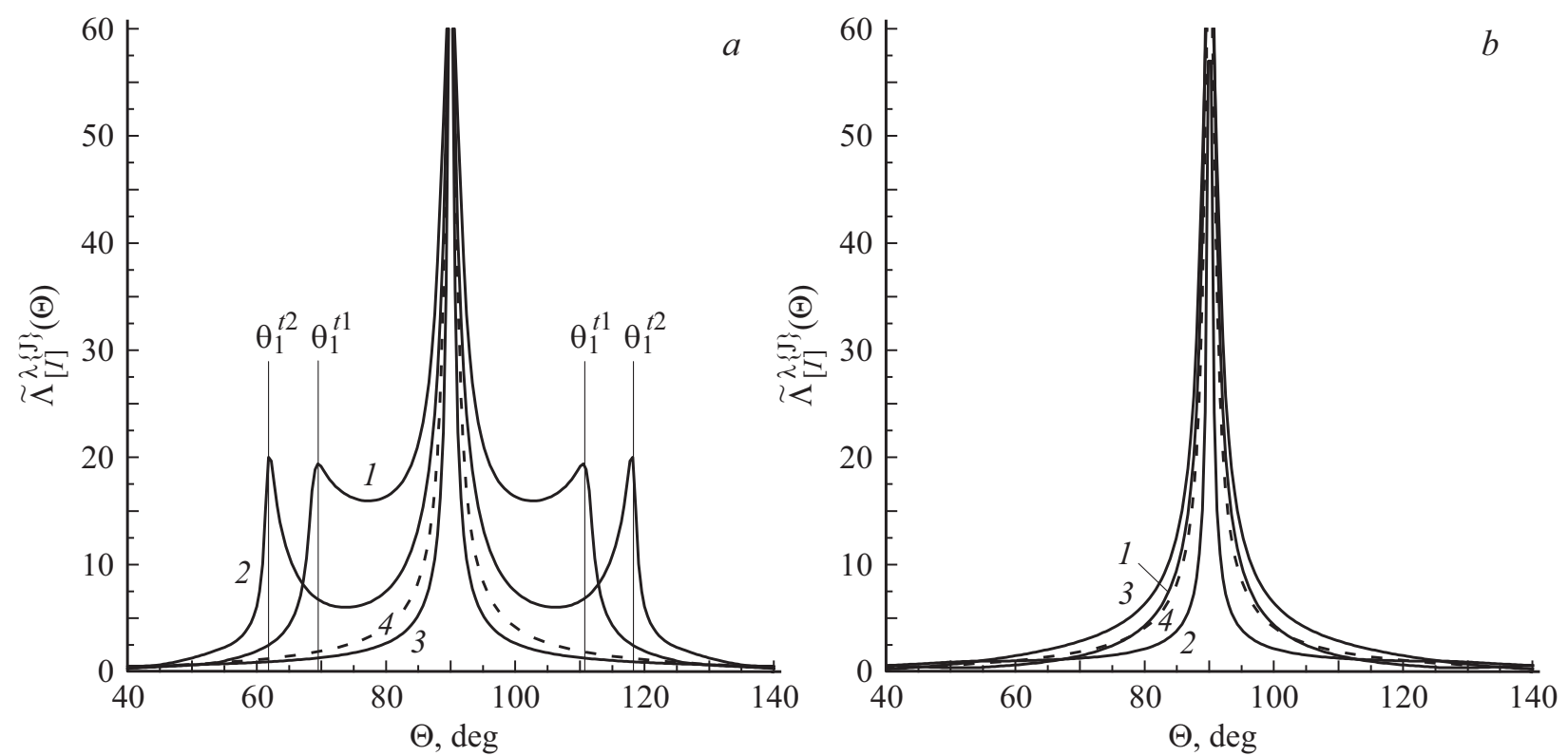

Рис. 2. Распределение длин пробега $\tilde{\Lambda}_{[I(\psi)]}^{\lambda\{J\}}(\Theta)$ в поперечном сечении для пленок $\mathrm{GaAs}(a)$ и $\mathrm{CaF}_{2}(b)$ с ориентацией $\{100\}$ и параметрами $L=W=100 D, D=50 \mathrm{~nm}$ : кривые 1 - для моды $t_{1}$, кривые 2 - для моды $t_{2}$, кривые 3 - для продольных фононов, кривая 4 - для модели изотропной среды.

дающих при усреднении длин пробега основной вклад, значительно сужаются. Поэтому при переходе к длинным пленкам $L \gg W$ усреднение по углам $\Phi$ в плоскостях этих пленок оказывается уже недостаточным, чтобы полностью размыть эффект фокусировки фононов. В результате длины пробега становятся анизотропными. Основной вклад в анизотропию теплопроводности и средних длин пробега фононов в пленках с ориентацией $\{100\}$ на основе кристаллов первого типа вносят медленные поперечные моды. Они фокусируются в направлениях [100] и их длины пробега в пленках GaAs оказываются на 53\% больше, чем в направлениях [110]. Однако в этих направлениях длины пробега $\Lambda_{[100]}^{\{100\} t 2}$ имеют локальные минимумы, а максимальных значений они достигают при углах $\Psi=n \pi / 2 \pm \theta_{3}$, и их величины в пленках GaAs оказываются на $10 \%$ меньше. Анизотропия теплопроводности и средних длин пробега в длинных пленах $(L \gg W)$ оказывается несколько меньше: отношение $\Lambda_{[100]}^{\{100\}}: \Lambda_{[110]}^{\{100\}}=1.21$. В пленках из кристаллов второго типа для ориентации $J=\{100\}$ основной вклад в теплопроводность вносит быстрая поперечная мода, которая фокусируется в секторе $\left[n \pi / 4-\theta_{3}, n \pi / 4+\theta_{3}\right]$ и обеспечивает максимум теплопроводности. Так, например, для пленок $\mathrm{CaF}_{2} \Lambda_{[110]}^{\{100\} t 1}: \Lambda_{[100]}^{\{100\} t 1}=1.35$, а $\Lambda_{[110]}^{\{100\}}: \Lambda_{[100]}^{\{100\}}=1.13$. Отметим, что в длинных пленках GaAs c ориентацией $\{100\}$ средние длины пробега фононов оказываются больше, а для ориентации $\{111\}-$ меньше, чем $\Lambda_{i s o}$. Тогда как в кристаллах второго типа ситуация обратная: для пленок с $J=\{100\}$ средние длины пробега фононов оказываются меньше, а для $J=\{111\}-$ больше, чем $\Lambda_{i s o}$. Итак, для длинных пленок из кристаллов первого и второго типа длины пробега фононов качественно отличаются. Отметим, что в длинных пленках с ориентацией $\{110\}$ эллипсоидальный вид средних длин пробега сохраняется.

\section{3. Влияние фокусировки на распространение фононных мод в квадратных пленках с ориентациями плоскостей $\{100\}$ и $\{111\}$}

Рассмотрим влияние фокусировки на распространение фононных мод. Для этого усредним длины пробега по углам $\Phi$ в плоскостях пленок и построим их зависимости от угла $\Theta$ в поперечном сечении пленки, которое включает направление теплового потока (см. рис. 2). Как видно из рис. 2, $а$, для поперечных фононов в пленках из кристаллов первого типа с ориентацией $\{100\}$ преобладает эффект фокусировки, и усредненные по углам $\Phi$ длины пробега $\Lambda_{[001]}^{t 1\{100\}}(\Theta)$ и $\Lambda_{[001]}^{t 2\{100\}}$ при всех углах $\Theta$ оказываются больше, чем $\Lambda_{\text {iso }}(\Theta)$, тогда как для продольных фононов - наоборот: их длины пробега при всех углах оказываются меньше, чем $\Lambda_{i s o}$. В кристаллах второго типа ситуация обратная: для медленной поперечной моды $\Lambda_{[001]}^{t 2\{100\}}$ гораздо меньше, чем $\Lambda_{i s o}(\Theta)$, а для $\Lambda_{[001]}^{t 1\{100\}}(\Theta)$ незначительно превышает значения $\Lambda_{i s o}(\Theta)$. Для продольных фононов преобладает эффект фокусировки и их длина пробега оказываются больше, чем $\Lambda_{i s o}$. Однако вклад продольных фононов в теплопроводность мал. Отсюда следует, средние длины 
пробега в пленках из кристаллов второго типа будут меньше, а из первого типа больше, чем $\Lambda_{i s o}$.

Следует отметить, что возникновение боковых максимумов на зависимостях $\Lambda_{[001]}^{t 2\{100\}}(\Theta)$ (см. рис. 2,a) обусловлено построением распределения длин пробега по поперечному сечению пленки в пространстве волновых векторов. В координатном пространстве (или пространстве групповых скоростей) они соответствуют волновым векторам с углами $\pm \theta_{1}^{t 2}$, групповая скорость которых совпадает с направлением [100], т.е. боковые максимумы перейдут в центральный пик, соответствующий направлению градиента температуры.

Для квадратных пленок с $J=\{111\}$ зависимости длин пробега $\Lambda_{[I(\psi)]}^{\lambda\{100\}}(\Theta)$ для всех акустических мод существенно изменяются (см. рис. 3). Центральный максимум сохраняется только для изотропной среды $\Lambda_{i s o}(\Theta)$, а для всех акустических мод зависимости длин пробега $\Lambda_{[I(\psi)]}^{\lambda\{100\}}(\Theta)$ в пленках из кристаллов обоих типов в окрестности направления теплового потока (оси пленки) имеют плавные минимумы (см. рис. 3). Это означает, что для всех мод в направлениях близких к направлению теплового потока преобладают эффекты дефокусировки, в отличие от пленок с ориентацией $\{100\}$. Причем, длины пробега $\Lambda_{\{111\}}^{2}(\Theta)$ в пленках из кристаллов первого типа $\mathrm{GaAs}$ почти во всем интервале углов $\Theta$ оказываются меньше, чем $\Lambda_{i s o}(\Theta)$, за исключением узких интервалов $84<\Theta<86^{\circ}$ и $930<\Theta<96^{\circ}$, где они имеют локальные максимумы. Очевидно, что среднее значение $\Lambda_{\{111\}}^{t 2}$ будет меньше, чем $\Lambda_{i s o}$. Для быстрой поперечной и продольной мод интервалы углов $\Theta$, где $\Lambda_{[I(\psi)]}^{\lambda\{111\}}(\Theta)>\Theta_{\text {iso }}(\Theta)$ значительно шире, поэтому средние значения для них будут незначительно выше, чем $\Lambda_{\text {iso }}$ (см. рис. 1). Однако при этом средняя длина свободного пробега для GaAs оказывается меньше, чем в модели изотропной среды.

Для пленок из кристаллов второго типа $\mathrm{CaF}_{2}$ боковые пики для быстрой поперечной моды оказываются значительно выше, чем для GaAs. Поэтому средние длины пробега $\Lambda_{\{111\}}^{t 1}$ в них оказываются почти в 1.7 раза больше, чем $\Lambda_{i s o}$, и в 1.5 раза больше, чем в GaAs. Для медленной поперечной и продольной мод интервалы углов $\Theta$, где $\Lambda_{[I(\psi)]}^{\lambda\{11\}}(\Theta)>\Lambda_{i s o}(\Theta)$ значительно уже, поэтому средние значения $\Lambda_{[I(\psi)]}^{\lambda\{11\}}$ для них оказываются меньше, чем $\Lambda_{i s o}$ (см. рис. 1). Доминирующая роль быстрой поперечной моды в пленках $\mathrm{CaF}_{2}$ приводит к тому, что средняя длина свободного пробега для них оказывается больше, чем в модели изотропной среды в 1.4 раза.

Из работ Фукса, Зондгеймера $[17,18]$ известно, что при достаточно низких температурах теплосопротивление пленок определяется, главным образом, рассеянием на плоскостях пленок. В теории Казимира предполагается, что все фононы при соударении с поверхностью поглощаются, а затем переизлучаются изотропно в полупространство по направлению внутрь образца.

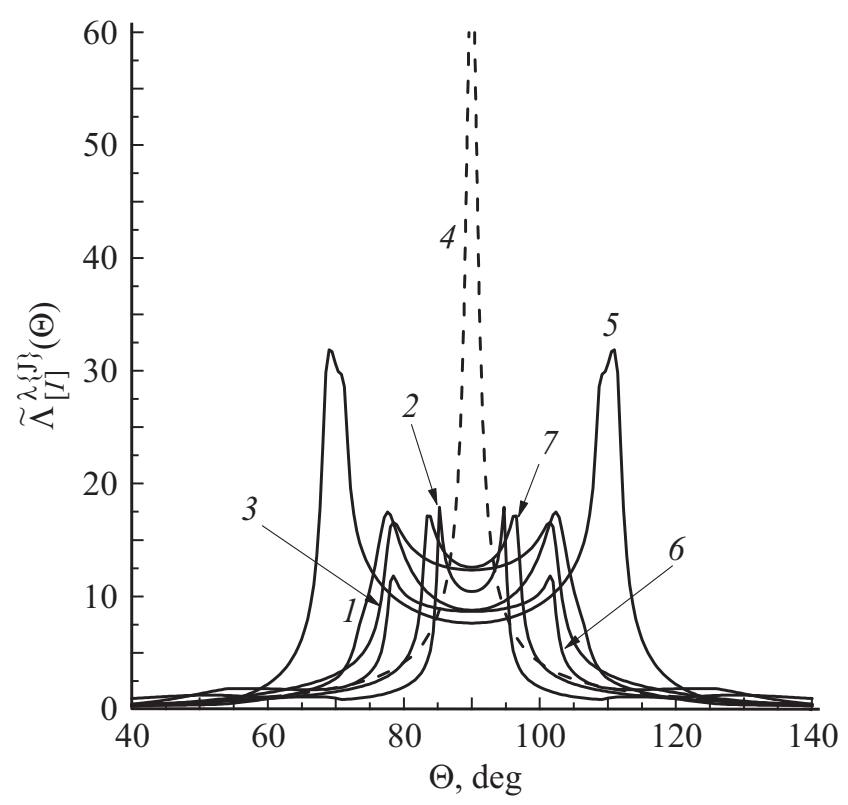

Рис. 3. Распределение длин пробега $\tilde{\Lambda}_{[I(\psi)]}^{\lambda\{J\}}(\Theta)$ в поперечном сечении для пленок GaAs (кривые 1,2,3) и $\mathrm{CaF}_{2}$ (кривые 5,6,7) с ориентацией $\{111\}$ и параметрами $L=W=100 D, D=50 \mathrm{~nm}$ : кривые 1,5 - для моды $t_{1}$, кривые 2,6 - для моды $t_{2}$, кривые 3,7 - для продольных фононов, кривая 4 - для модели изотропной среды.

Поэтому в каждой точке поверхности независимо от ее ориентации и параметров анизотропии $k-1$ фононы всех поляризаций рассеиваются диффузно. Таким образом, рассеивающая способность или интенсивность рассеяния пленок с различной ориентацией плоскостей одинакова. Поэтому предположение о различной „рассеивающей способности“ плоскостей пленок с различной ориентацией в [19] для характеристики влияния фокусировки фононов на их теплопроводность пленок является физически некорректным. Итак, мы показали, что влияние фокусировки фононов на теплопроводность пленок связано с отличием направления распространения фононов и направления волнового вектора и обусловлено упругой анизотропией кристаллов. Если направление теплового потока и ось пленки совпадает с направлением фокусировки одной из мод, то направления распространения фононов этой моды будут отклоняться от плоскостей пленки к ее оси и длина пробега этих фононов будет возрастать, как это имеет место для медленных поперечных фононов в пленках с ориентацией $\{100\}$ и направлением потока тепла [100].

\section{4. Заключение}

Исследовано влияние фокусировки фононов на распространение фононов и фононный транспорт в пленках с различным типом анизотропии упругой энергии. Oc- 
новные результаты исследований могут быть сформулированы следующим образом:

1. Установлены физические причины, приводящие к изотропной зависимости теплопроводности для квадратных пленок из кристаллов обоих типов с ориентациями $\{100\}$ и $\{111\}$. Показано, что анизотропия длин свободного пробега в квадратных пленках обусловлена их зависимостью от ориентации плоскости. Для кристаллов первого и второго типа она качественно отличается: максимальные значения теплопроводности из кристаллов первого типа достигаются для ориентации $\{001\}$, а минимальные - для $\{111\}$. Однако в пленках из кристаллов второго типа максимальные значения достигаются для $J=\{111\}$, а минимальные - для $J=\{001\}$.

2. Для квадратных пленок с ориентацией $\{110\}$ угловые зависимости длин пробега фононов в пленках из кристаллов разного типа также качественно отличаются: они имеют эллипсоидальный вид, но в кристаллах первого типа длинная ось эллипсоида направлена вдоль [100], а короткая - вдоль $[110]$; тогда, как для кристаллов второго типа наоборот — длинная ось эллипсоида вдоль [110], а короткая - вдоль [100].

3. Анализ распределения длин пробега в поперечном сечении квадратных пленок показал, что теплопроводность и средние длины пробега фононов в пленках с ориентацией $\{100\}$ из кристаллов первого типа имеют большие значения, чем для пленок из кристаллов второго типа, поскольку для первых преобладает эффект фокусировки, тогда как для вторых - эффект дефокусировки фононов. Однако в пленках с ориентацией $\{111\}$ из кристаллов второго типа средние длины пробега фононов оказываются больше, чем для пленок из кристаллов первого типа.

\section{Список литературы}

[1] D.G. Cahill, W.K. Ford, K.E. Goodson, G.D. Mahan, A. Majumdar, H.J. Maris, R. Merlin, S.R. Phillpot. J. Appl. Phys. 93, 793 (2003).

[2] A.D. McConnell, K.E. Goodson. Ann. Rev. Heat Transfer. 14, 129 (2005).

[3] D. Li, Y. Wu, P. Kim, L. Shi, P. Yang, A. Majumdar. Appl. Phys. Lett. 83, 2934 (2003).

[4] M. Asheghi, Y.K. Leung, S.S. Wong, K.E. Goodson. Appl. Phys. Lett. 71, 1798 (1997); M. Asheghi, M.N. Touzelbaev, K.E. Goodson, Y.K. Leung, S.S. Wong. J. Heat Transfer. 120, 30 (1998).

[5] D.G. Cahill, P.V. Braun, G. Chen, D.R. Clarke, S. Fan, K.E. Goodson, P. Keblinski, W.P. King, G.D. Mahan, A. Majumdar, H.J. Maris, S.R. Phillpot, E. Pop, L. Shi. J. Appl. Phys. Rev. 1, 011305 (2014).

[6] H.J. Maris, S. Tamura. Phys. Rev. B. 85, 054304 (2012).

[7] И.Г. Кулеев, И.И. Кулеев, С.М. Бахарев. ЖЭТФ 145, 292 (2014).

[8] И.И. Кулеев, С.М. Бахарев, И.Г. Кулеев, В.В. Устинов. ЖЭТФ 147, 736 (2015).

[9] И.Г. Кулеев, И.И. Кулеев. ФТТ 49, 422 (2007).
[10] B. Truel, C. Elbaum, B.B. Chick. Ultrasonic methods in sold state physics. Academic press. N.Y.-London (1969). 464 p.

[11] J.R. Drabble, A.J. Brammer. Solid State Commun. 4, 467 (1966).

[12] C.V. Briscoe, C.F. Squire. Phys. Rev. 106, 1175 (1957).

[13] H.J. McSkimin, Andreatch. J. Appl. Phys. 35, 2161 (1964).

[14] H.J. McSkimin, W.L. Bond. Phys. Rev. 105, 116 (1957).

[15] И.И. Кулеев, И.Г. Кулеев, С.М. Бахарев. ЖЭТФ 146, 522 (2014).

[16] И.И. Кулеев. ФТТ 59, 668 (2017).

[17] K. Fuchs. Proc. Cambridge Philos. Soc. 34, 100 (1938).

[18] E.H. Sondheimer. Adv. Phys. 1, 1 (1952).

[19] Z. Aksamija, I. Knezevic. Phys. Rev. B 82, 045319 (2010). 